\title{
The management of non-muscle-invasive bladder cancer: A comparison of European and UK guidelines
}

\author{
A Leiblich ${ }^{1,2}$, RJ Bryant ${ }^{1,2}, \mathrm{R}$ McCormick ${ }^{2}$ and J Crew ${ }^{2}$
}

\begin{abstract}
Bladder cancer represents a significant health burden worldwide, with non-muscle-invasive tumours representing the majority of new bladder cancer diagnoses. Non-muscle-invasive bladder cancer (NMIBC) has a high prevalence and forms a large proportion of the caseload in urological practice, accounting for a significant cost in terms of urologic al healthcare. The last two decades have seen the development and refinement of guidelines from national and international organisations aiming to optimise management of NMIBC and bladder cancer in general. This review outlines the most recent European and United Kingdom guidelines on NMIBC, and in particular focuses on comparing and contrasting key recommendations from guidelines published by the European Association of Urology and the UK-based National Institute for Clinical Excellence.
\end{abstract}

Level of evidence: Not applicable for this multicentre audit.

\section{Keywords}

Bladder cancer, superficial, NMIBC, guidelines

\section{Introduction}

Bladder cancer is the seventh commonest cancer worldwide to affect men, and the 14th commonest cancer to affect women. Rates of bladder cancer appear to be higher in the developed world, where it represents the fourth commonest cancer in men. ${ }^{1}$ The United Kingdom (UK) has the lowest incidence of bladder cancer in men in Europe and the 14th lowest for women. ${ }^{2}$ Bladder cancer accounts for $3 \%$ of all cancer deaths in the UK, with a crude mortality rate of 8.2 cancer deaths per 100,00 individuals. ${ }^{3}$

Most (75\%-80\%) patients with bladder cancer present with disease confined to the mucosa. Non-muscle-invasive bladder cancer (NMIBC) has a higher prevalence than muscle-invasive tumours and a lower risk of cancer-specific mortality., 2,45 Management of NMIBC is therefore a common and significant feature of secondary care urological practice.

This review outlines the most recent UK and European guidelines regarding the management of NMIBC, with a particular emphasis on comparing and contrasting

European Association of Urology (EAU) ${ }^{6}$ and National Institute of Clinical Excellence (NICE) ${ }^{7}$ guidelines.

\section{Diagnosis and initial evaluation of NMIBC}

There is consensus between the EAU and NICE guidance regarding initial evaluation of patients presenting with suspected bladder cancer. Both guidelines stress the

1 Nuffield Department of Surgical Sciences, University of Oxford, Level 6, John Radcliffe Hospital, UK

2Department of Urology, Churchill Hospital, Oxford University

Hospitals NHS Foundation Trust, UK

\section{Corresponding author:}

Jeremy Crew, Department of Urology, Churchill Hospital, Oxford University Hospitals NHS Foundation Trust, Headington, Oxford, UK. Email: Jeremy.Crew@ouh.nhs.uk 
importance of taking a comprehensive patient history and recommend cystoscopy for all patients with symptoms suggestive of bladder cancer. Both guidelines also suggest that the evidence for using urinary biomarkers in this setting is inadequate and they should not be substituted for cystoscopy to investigate suspected bladder cancer or for follow-up after treatment.

The EAU NMIBC guidelines suggest that a renal and bladder ultrasound may be valuable in the work-up of patients presenting with haematuria, but once a diagnosis of NMIBC is made computed tomography urography (CTU) should be performed only in selected cases (e.g. tumours located in the trigone, or multiple and/or high-risk tumours). This strategy is based on the observation that once a bladder tumour has been detected, the incidence of further significant findings on $\mathrm{CT}$ is low. The overall incidence of upper tract urothelial cancer is only about $1.8 \%$ in patients with newly diagnosed bladder cancer, although this increases to $7.5 \%$ in patients with trigone tumours. In contrast, NICE guidance recommends either CT or magnetic resonance imaging prior to transurethral resection of bladder tumour (TURBT) only if muscle-invasive disease is suspected at initial cystoscopy. In practice however it is commonplace for many patients in the UK to receive upper tract imaging as part of their initial assessment for haematuria.

EAU guidelines advocate collection of urine for cytological analysis to predict high-grade tumour before TURBT. This differs from NICE guidance, which suggests patients with suspected bladder cancer should be offered white light-guided TURBT along with photodynamic diagnosis, narrow-band imaging, urinary cytology or a urinary biomarker test such as UroVysion, ImmunoCyt or the nuclear matrix protein 22 test. Regarding urinary molecular markers (as opposed to cytology) the EAU guidelines comment that currently available tests are not sufficiently robust for routine clinical practice, because whilst many display greater sensitivity than cytology for detecting bladder cancer, they have lower specificity.

\section{TURBT for NMIBC}

In contrast to UK NICE guidelines, European guidelines provide a thorough step-wise description of optimum TURBT technique. NICE guidelines simply state that detrusor muscle should be obtained during TURBT, that the size and number of tumours should be recorded, and that random biopsies should be taken only if there is a specific clinical indication, e.g. investigation of positive urine cytology. EAU guidance concurs with these recommendations, but provides additional guidance regarding TURBT. This additional guidance includes recommendation for bimanual palpation under anaesthesia, which is not mentioned in NICE guidance. Furthermore, EAU guidelines recommend biopsy of the prostatic urethra in cases of bladder neck tumour, when bladder carcinoma in situ (CIS) is present, or when there is positive cytology without obvious bladder tumour. ${ }^{8} 9$ These recommendations are absent from NICE guidelines.

Both EAU and NICE guidelines recommend that a single dose of intravesical Mitomycin C should be given as soon as possible after initial TURBT, based on strong evidence that this is associated with a significant reduction in tumour recurrence. Three large meta-analyses have demonstrated that postoperative intravesical chemotherapy after TURBT reduces the risk of recurrence from $24.7 \%$ to $13.0 \%$ compared with TURBT alone.8,10-12

\section{Risk stratification of NMIBC}

Tumours should be stratified into low-, intermediate- and high-risk groups based on histological analysis, tumour size and number. Such risk stratification provides prognostic information and guides ongoing management. The precise criteria used for risk stratification are similar between sets of guidelines (Table 1), although one important difference is the inclusion within the intermediate-risk group of the NICE guidance of 'any recurrent low-risk NMIBC recurring within 12 months of the last tumour occurrence'. This stipulation does not exist within EUA guidelines, and hence early recurrences would not necessarily be classified as intermediate risk.

\section{Re-do TURBT}

NICE and EAU guidelines assess the value of repeat TURBT within six weeks due to the risk of residual tumour after initial TURBT..$^{13}$ Guidance is consistent that repeat TURBT should be performed if muscle is absent from the initial TURBT specimen (except CIS and low-grade Ta). However, there remain subtle differences between NICE and EAU guidelines regarding criteria that mandate repeat TURBT. For instance, NICE recommend repeat TURBT on all NMIBC patients with high-risk tumours, whereas EAU guidance advocates re-resection in all T1 tumours and all high-grade lesions. ${ }^{14-16}$ According to NICE risk stratification (see Table 1), G2pTa (high-grade) tumours are intermediate risk and thus repeat TURBT is not necessary, whereas EAU guidelines advocate re-resection of all high-grade lesions (except CIS). Both guidelines assert that repeat TURBT should be performed within six weeks of the initial resection.

\section{Disease management}

Additional treatment for NMIBC after initial TURBT depends on tumour risk-stratification, with treatment options ranging from single-dose intravesical chemotherapy to radical cystectomy (RC). EAU treatment recommendations for NMIBC are summarised in Table 2. NICE 
Table 1. Risk stratification of NMIBC according to EAU and NICE guidelines.

\begin{tabular}{|c|c|c|}
\hline Risk group stratification & EAU guidelines & NICE guidelines \\
\hline Low-risk tumours & $\begin{array}{l}\text { Primary, solitary, Ta, G1 } \\
\text { (PUNLMP, LG), }<3 \mathrm{~cm} \text {, no CIS }\end{array}$ & $\begin{array}{l}\text { Solitary pTaG } 1<3 \mathrm{~cm} \text {, solitary pTaG } 2(\mathrm{LG})<3 \\
\mathrm{~cm} \text {, PUNLMP }\end{array}$ \\
\hline $\begin{array}{l}\text { Intermediate-risk } \\
\text { tumours }\end{array}$ & $\begin{array}{l}\text { All tumours not defined in the two } \\
\text { adjacent categories }\end{array}$ & $\begin{array}{l}\text { Any cancer that is not high or low risk, including: } \\
\text { - } \text { solitary } \mathrm{pTaG} 1>3 \mathrm{~cm} \\
\text { - } \text { multifocal } \mathrm{pTaG} 1 \\
\text { - } \text { solitary } \mathrm{pTaG}(\mathrm{LG})>3 \mathrm{~cm} \\
\text { - } \text { multifocal pTaG2 (high grade) } \\
\text { - } \text { pTaG2 (high grade) } \\
\text { - Any low-risk NMIBC recurring within } 12 \\
\text { months of last tumour occurrence }\end{array}$ \\
\hline High-risk tumours & $\begin{array}{l}\text { Any of the following: } \\
\text { - } \quad \mathrm{T} 1 \text { tumour } \\
\text { - } \mathrm{G} 3 \text { (high-grade) tumour } \mathrm{CIS} \\
\text { - } \text { Multiple and recurrent and } \\
\quad \text { large }(>3 \mathrm{~cm}) \mathrm{TaG} 1 / 2 \text { tumours }\end{array}$ & $\begin{array}{l}\text { Urothelial cancer with any: } \\
\square \text { pTaG3 } \\
\square \text { pT1G2 } \\
\text { pT1G3 } \\
\text { pTis (CIS) } \\
\text { - Aggressive variants of urothelial carcinoma, for } \\
\text { example. micropapillarv or nested variants }\end{array}$ \\
\hline
\end{tabular}

CIS: carcinoma in situ; EAU: European Association of Urology; LG: low-grade; NICE: National Institute of Clinical Excellence; NMIBC: non-muscle-invasive bladder cancer; PUNLMP: papillary urothelial neoplasm of low malignant potential.

Table 2. EAU treatment recommendations for NMIBC according to risk stratification.

\begin{tabular}{|c|c|c|}
\hline Risk category & Definition & Treatment recommendation \\
\hline Low-risk & Primary, solitary, Ta, LG/G1, <3 cm, no CIS & $\begin{array}{l}\text { One immediate instillation of chemotherapy } \\
\text { post-TURBT }\end{array}$ \\
\hline Intermediate-risk & $\begin{array}{l}\text { All cases between categories of low and high } \\
\text { risk }\end{array}$ & $\begin{array}{l}\text { One immediate instillation of chemotherapy } \\
\text { followed by further instillations, with } \\
\text { chemotherapy for a maximum of one year or one } \\
\text { year full-dose BCG }\end{array}$ \\
\hline High-risk & $\begin{array}{l}\text { T1 tumours; HG/G3 tumours; CIS; multiple } \\
\text { and recurrent and large }(>3 \mathrm{~cm}) \text { Ta } \mathrm{G} 1 / 2 \\
\text { tumours }\end{array}$ & $\begin{array}{l}\text { Intravesical full-dose BCG instillations for one } \\
\text { to three years or cystectomy (in highest-risk } \\
\text { tumours) }\end{array}$ \\
\hline $\begin{array}{l}\text { Subgroup of highest } \\
\text { risk tumours }\end{array}$ & $\begin{array}{l}\text { G3T1 associated with concurrent CIS; } \\
\text { multiple and/or large G3T1 and/or recurrent } \\
\text { G3T1; G3T1 with CIS in prostatic urethra/ } \\
\text { unusual histology of urothelial carcinoma/ } \\
\text { lympho-vascular invasion; BCG failures }\end{array}$ & $\begin{array}{l}\text { Radical cystectomy }(\mathrm{RC}) \text { should be considered for } \\
\text { all patients; For patients who refuse or are } \\
\text { unsuitable for RC, intravesical full-dose BCG } \\
\text { installations for one to three years; RC is } \\
\text { recommended for BCG failures }\end{array}$ \\
\hline
\end{tabular}

CIS: carcinoma in situ; EAU: European Association of Urology; BCG: Bacillus Calmette-Guerin; LG: low-grade; NMIBC: non-muscle-invasive bladder cancer; TURBT: transurethral resection of bladder tumour.

and EUA guidance are very similar regarding disease management, with both recommending that treatment should be based on risk-stratification. Low-risk tumours require no further treatment beyond a single dose of Mitomycin $\mathrm{C}$ following initial TURBT. NICE guidance recommends that patients with intermediate-risk NMIBC should be offered a six-dose course of intravesical Mitomycin $\mathrm{C}$, but these recommendations do not mention any role for Bacillus Calmette-Guerin (BCG) therapy in the management of intermediate-risk disease. In contrast the EAU guidance advocates either intravesical chemotherapy for up to a year or a one-year course of BCG therapy. Both NICE and EAU guidance recommend offering either BCG therapy (for one to three years) or RC to patients with high-risk disease. A key difference between EAU and NICE guidelines is that, unlike the EAU guidelines, NICE does not sub-stratify high-risk disease to include a subgroup of highest risk tumours for whom RC should strongly be considered over BCG. The choice of treatment should be based on a full discussion with the patient, 
covering advantages and disadvantages of each treatment modality and the risk of progression.

\section{Follow-up of patients with NMIBC}

Given the risk of recurrence and progression in patients with NMIBC, regular follow-up with cystoscopy is mandated by both EAU and NICE guidelines. In general there is agreement that patients with NMIBC require regular cystoscopy surveillance to check for intravesical recurrence, but the optimal frequency and length of follow-up is contentious, and NICE and EAU guidelines differ with respect to follow-up of low-risk NMIBC. The risk of recurrence or progression of NMIBC may be predicted through use of risk tables, and cystoscopy follow-up strategies may be tailored accordingly to enable follow-up to be safely reduced for low-risk groups whilst ensuring that high-risk groups are monitored closely. ${ }^{17,18}$ Whilst both advise cystoscopy at three months post-TURBT and a further cystoscopy at 12 months postTURBT, if the cystoscopy at 12 months demonstrates no recurrence then NICE recommends discharge back to primary care. This particular guidance is based on evidence suggesting that if a low-risk NMIBC tumour recurs after the first year then it typically does so with the same low-risk stage and grade, and the patient would usually present with recurrent symptoms such as haematuria. However, this aspect of NICE guidance regarding discharge to primary care differs markedly from EAU recommendations, which advise annual-check cystoscopies for five years before discharge. There is no clear consensus between the EAU and NICE guidelines regarding the timing of discharge from follow-up for patients with lowrisk disease, although the literature suggests that the risk of progression is low and that most recurrences are low grade and non-life-threaten-ing. ${ }^{19-22}$ The available evidence also indicates that the risk of recurrence after five recurrence-free years is low, ${ }^{23}$ suggesting that discharge at five years can be considered safe.

NICE guidelines advise cystoscopy follow-up for patients with intermediate-risk NMIBC at a schedule of three, nine and 18 months, with annual-check cystoscopies thereafter. Discharge to primary care for this group of patients can be considered after five years of disease-free follow-up. This contrasts with EAU guidance, which simply recommends a follow-up schedule for patients with intermediate-risk Ta tumours to be somewhere between the surveillance schedule for low- and high- risk disease, with scheduling adapted according to personal and subjective factors.

The follow-up of high-risk NMIBC differs between the two sets of guidelines. NICE recommend check cystosco-pies at a schedule of every three months for the first two years, then every six months for the next two years, and annually thereafter. In contrast, EAU guidance recommends cystoscopy and urine cytology at three-monthly intervals for the first two years and then every six months thereafter for five years, followed by annual-check cystoscopy. EAU guidance also advises annual upper tract imaging (either CTU or intravenous urogram) for high-risk tumours, whereas NICE guidance does not mention this.

\section{Conclusion}

The EAU and NICE guidelines for NMIBC provide considerable consensus regarding the management of this common malignancy, which is often difficult to manage. However, there are key differences between the guidelines, and there are a small number of areas that are open to interpretation. These guidelines have been developed in order to optimise the management of patients with NMIBC and equip urologists with a set of standards with which to guide their practice. We recommend that urologists actively involved in the management of NMIBC should be familiar with both sets of guidelines and incorporate their key recommendations into their clinical practice.

\section{Acknowledgements}

None.

\section{Conflicting interests}

The Authors declare that there is no conflict of interest.

\section{Funding}

This research received no specific grant from any funding agency in the public, commercial, or not-for-profit sectors.

\section{Ethical approval}

Not applicable.

\section{Informed consent}

Not applicable.

\section{Guarantor}

AL.

\section{Contributorship}

$\mathrm{AL}$ researched literature and wrote the first draft of the manuscript. RJB and RM contributed further text and literature research. JC oversaw the project and developed the final draft with AL, RJB and RM.

\section{References}

1. Jemal A, Bray F, Center MM, et al. Global cancer statistics. CA Cancer J Clin 2011; 61: 69-90.

2. Burger M, Catto JW, Dalbagni G, et al. Epidemiology and risk factors of urothelial bladder cancer. Eur Urol 2013; 63: 234-241.

3. Cancer Research UK. Bladder cancer statistics. 2013. http:// www.cancerresearchuk.org/health-professional/cancerstatistics/statistics-by-cancer-type/bladder-cancer (accessed 16 June 2016) 
4. Bosetti C, Bertuccio P, Chatenoud L, et al. Trends in mortality from urologic cancers in Europe, 1970-2008. Eur Urol 2011; 60: 1-15.

5. Chavan S, Bray F, Lortet-Tieulent J, et al. International variations in bladder cancer incidence and mortality. Eur Urol 2014; 66: 59-73.

6. Babjuk M, Böhle A, Burger M, et al. EAU Guidelines on non-muscle-invasive urothelial carcinoma of the bladder: Update 2016. Eur Urol 2017; 71: 447-461.

7. National Institute for Health and Care Excellence. Bladder cancer: Diagnosis and management. 2015. https://www.nice. org.uk/guidance/ng2/resources/bladder-cancer-diagnosisand-management-pdf-51036766405 (accessed 3 June 2016).

8. van der Meijden A, Oosterlinck W, Brausi $M$, et al. Significance of bladder biopsies in Ta,T1 bladder tumors: A report from the EORTC Genito-Urinary Tract Cancer Cooperative Group. EORTC-GU Group Superficial Bladder Committee. Eur Urol 1999; 35: 267-271.

9. Hara T, Takahashi M, Gondo T, et al. Risk of concomitant carcinoma in situ determining biopsy candidates among primary non-muscle-invasive bladder cancer patients: Retrospective analysis of 173 Japanese cases. Int J Urol 2009; 16: 293-298.

10. Abern MR, Owusu RA, Anderson MR, et al. Perioperative intravesical chemotherapy in non-muscle-invasive bladder cancer: A systematic review and meta-analysis. J Natl Compr Canc Netw 2013; 11: 477-484.

11. Sylvester RJ, Oosterlinck W and van der Meijden AP. A single immediate postoperative instillation of chemotherapy decreases the risk of recurrence in patients with stage Ta T1 bladder cancer: A meta-analysis of published results of randomized clinical trials. J Urol 2004; 171 (6 Pt 1): 21862190, quiz 2435.

12. Perlis N, Zlotta AR, Beyene J, et al. Immediate post-transurethral resection of bladder tumor intravesical chemotherapy prevents non-muscle-invasive bladder cancer recurrences: An updated meta-analysis on 2548 patients and quality-ofevidence review. Eur Urol 2013; 64: 421-430.

13. Brausi M, Collette L, Kurth K, et al. Variability in the recurrence rate at first follow-up cystoscopy after TUR in stage Ta T1 transitional cell carcinoma of the bladder:
A combined analysis of seven EORTC studies. Eur Urol 2002; 41: 523-531.

14. Grimm MO, Steinhoff C, Simon X, et al. Effect of routine repeat transurethral resection for superficial bladder cancer: A long-term observational study. J Urol 2003; 170: 433437.

15. Divrik RT, Yildirim U, Zorlu F, et al. The effect of repeat transurethral resection on recurrence and progression rates in patients with $\mathrm{T} 1$ tumors of the bladder who received intravesical mitomycin: A prospective, randomized clinical trial. J Urol 2006; 175: 1641-1644.

16. Divrik T, Yildirim U, Eroğlu AS, et al. Is a second transurethral resection necessary for newly diagnosed pT1 bladder cancer? J Urol 2006; 175: 1258-1261.

17. Sylvester RJ, van der Meijden AP, Oosterlinck W, et al. Predicting recurrence and progression in individual patients with stage Ta T1 bladder cancer using EORTC risk tables: A combined analysis of 2596 patients from seven EORTC trials. Eur Urol 2006; 49: 466-465; discussion 475-477.

18. Fernandez-Gomez J, Madero R, Solsona E, et al. Predicting nonmuscle invasive bladder cancer recurrence and progression in patients treated with bacillus Calmette-Guerin: The CUETO scoring model. J Urol 2009; 182: 2195-2203.

19. Holmang S, Andius P, Hedelin H, et al. Stage progression in Ta papillary urothelial tumors: Relationship to grade, immunohistochemical expression of tumor markers, mitotic frequency and DNA ploidy. J Urol 2001; 165: 1124-1128; discussion 1128-1130.

20. Borhan A, Reeder JE, O'Connell MJ, et al. Grade progression and regression in recurrent urothelial cancer. J Urol 2003; 169: 2106-2109.

21. Gofrit ON, Pode D, Lazar A, et al. Watchful waiting policy in recurrent Ta G1 bladder tumors. Eur Urol 2006; 49: 303306; discussion 306-307.

22. Soloway MS, Bruck DS and Kim SS. Expectant management of small, recurrent, noninvasive papillary bladder tumors. $J$ Urol 2003; 170: 438-441.

23. Mariappan P and Smith G. A surveillance schedule for G1Ta bladder cancer allowing efficient use of check cys-toscopy and safe discharge at 5 years based on a 25 -year prospective database. J Urol 2005; 173: 1108-1111. 